\title{
Estructura y variabilidad de la región organizadora de nucléolos en Prochilodus lineatus (Pisces, Prochilodontidae)
}

\author{
Caramello, C.S.; Cowper Coles, F.; Sánchez, S.; Jorge, L.C. \\ Instituto de Ictiología del Nordeste, Facultad de Ciencias Veterinarias, Universidad Nacional del Nordeste, \\ Sargento Cabral 2139, Corrientes (3400), Argentina. Tel/fax 03783-425753. E-mail: liliancj@vet.unne.edu.ar
}

\begin{abstract}
Resumen
Caramello, C.S.; Cowper Coles, F.; Sánchez, S.; Jorge, L.C. Estructura y variabilidad de la región organizadora de nucléolos en Prochilodus lineatus (Pisces, Prochilodontidae). Rev. vet. 22: 2, 123-126, 2011. Los peces de la familia Prochilodontidae se distribuyen en varias cuencas hidrográficas de América del Sur. El género Prochilodus presenta una de las distribuciones más amplias, encontrándose desde el Orinoco hasta el río de La Plata. En la Argentina es el recurso íctico más abundante del río Paraná y actualmente ha crecido el interés por esta especie. Dada su importancia económica, el objetivo del presente trabajo fue establecer su estructura cromosómica en ejemplares provenientes del río Paraná en la Provincia de Corrientes (Argentina), con especial énfasis al análisis de las regiones organizadoras de nucléolos (NORs). El estudio de los cromosomas mitóticos y de las NORs se realizó a través de la coloración convencional con Giemsa y nitrato de plata, respectivamente. El cariotipo de la especie está constituido por 54 cromosomas (meta/submetacéntricos), siendo observada la ocurrencia de microcromosomas $\mathrm{B}$, los cuales varían en número a nivel intra e interindividual, generando la ocurrencia de polimorfismos. La NOR está localizada en posición intersticial en el brazo largo de un par metacéntrico, presentando variaciones en cuanto a la posición y tamaño de las regiones Ag-NORs+, a saber: a) la NOR se presenta única y de igual tamaño en los dos homólogos, b) NOR grande ocupando la mitad del brazo largo y única en uno de los cromosomas; pequeña y única en el otro, c) doble región NOR en uno de los cromosomas y NOR única y pequeña en su homólogo. La diferencia de tamaño de la NOR es debida a alteraciones cromosómicas estructurales como duplicaciones, deleciones y crossing over desigual. La doble NOR probablemente sea el resultado de una inversión paracéntrica.
\end{abstract}

Palabras clave: pez, Prochilodus lineatus, citogenética, NOR, río Paraná, Argentina.

\begin{abstract}
Caramello, C.S.; Cowper Coles, F.; Sánchez, S.; Jorge, L.C. Structure and variability of nucleolus organization regions in Prochilodus lineatus (Pisces, Prochilodontidae). Rev. vet. 22: 2, 123-126, 2011. Fishes of Prochilodontidae family are distributed in several river basins of South America. The genus Prochilodus has one of the most widely distribution, since it is found from the Orinoco to the Río de la Plata rivers. In Argentina is the most abundant fishery resource of the Paraná River. Today the interest in this fish farming has increased. Due to its economic importance, the objective of this assay was to establish the chromosome structure of specimens from the Paraná River in Corrientes Province (Argentina), with emphasis on the analysis of nucleolus organizing regions (NORs). The study of mitotic chromosomes and the NORs were performed using conventional Giemsa staining and silver nitrate, respectively. The karyotype of the species consists of 54 chromosomes (meta /submetacentric), the occurrence of microchromosomes B can be observed, which vary in number at intraand interindividual level causing the occurrence of polymorphisms. The NOR is located in interstitial position on the long arm of a metacentric pair. Variations are found in the position and size of the regions Ag-NORs with the following description: a) the NOR presents unique and the same size in two homologous, b) Large and unique NOR occupying only half of the big arm, in one chromosome; small and unique in the other, c) double NOR region in one chromosome and a single small NOR in the homologous. The differences in size of the NORs are due to structural chromosomal abnormalities such as duplications, deletions and unequal crossing over. The double NOR is probably the result of a paracentric inversion.
\end{abstract}

Key words: fish, Prochilodus lineatus, cytogenetic, NOR, Paraná River, Argentina. 


\section{INTRODUCCIÓN}

El género Prochilodus tiene una amplia distribución en América del Sur ${ }^{17}$. Prochilodus lineatus, conocido como curimbatá, bocachico o sábalo, representa en la Argentina el recurso íctico más abundante del río Paraná; es un pez iliófago como las demás especies de la familia Prochilodontidae. Es considerado una especie importante desde el punto de vista económico por la facilidad de ser cultivado en ríos, estanques y represas, por su elevado tenor proteico y perfecto balance de aminoácidos esenciales ${ }^{18}$.

Desde el punto de vista citogenético el género Prochilodus fue inicialmente estudiado en cuencas hidrográficas de Brasil. El análisis cariotípico de las ocho especies investigadas reveló una gran similitud en la macroestructura cariotípica tanto a nivel morfológico como numérico ( $2 \mathrm{n}=54$ cromosomas). Estos resultados llevaron a concluir que se trata de un género con una evolución cariotípica predominantemente conservativa 23,24 .

El análisis de las regiones organizadoras de nucleolos (NORs) en Prochilodus, utilizando coloración con nitrato de plata, evidenció en la mayoría de las especies comparadas la ocurrencia de dos cromosomas portadores de las NORs, localizadas en la constricción secundaria del brazo largo del par cromosómico $\mathrm{n}^{\circ} 2$. Por otro lado, Prochilodus marggravii y Prochilodus affinis mostraron un cromosoma no identificado con NOR activa en la región telomérica ${ }^{24}$.

En peces, las regiones organizadoras de nucleolos han sido estudiadas extensamente, mostrándose como buenos marcadores genéticos, auxiliando estudios citotaxonómicos de especies relacionadas ${ }^{11}$. Estas regiones cromosómicas han mostrado una gran variabilidad de comportamiento en los diferentes grupos, no solamen-

A
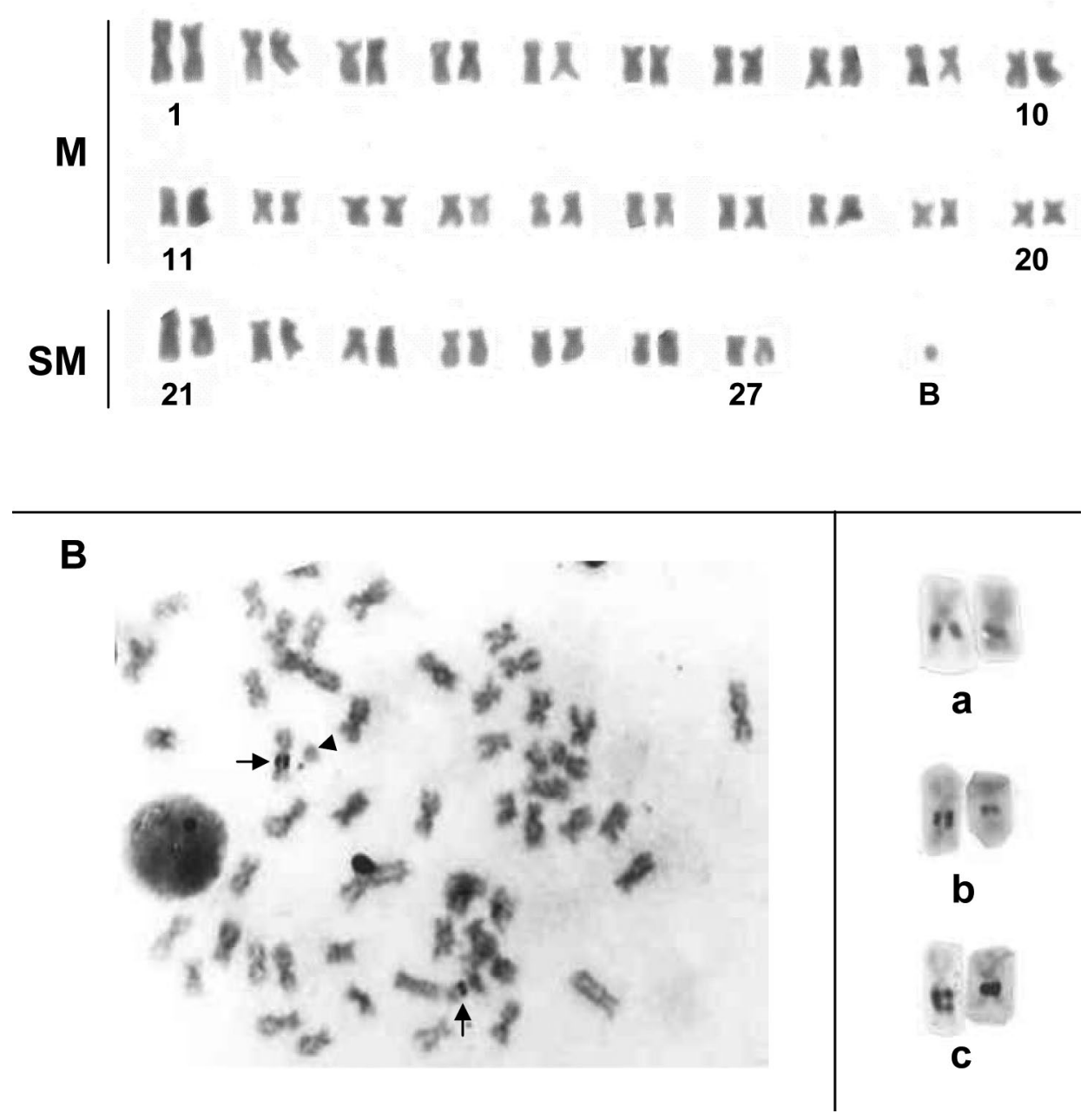

Figura 1. (A) Cariotipo de Prochilodus lineatus con un cromosoma B pequeño. (B) Metafase somática después de la coloración con nitrato de plata donde se observan los cromosomas portadores de la NOR (flechas) y un microcromosoma B (punta de flecha). En destaque a la derecha de la metafase, están evidenciados los pares portadores de los cistrons ribosómicos de diferentes individuos. 
te en relación al número, localización y tamaño de los cistrons ribosómicos, sino también en lo concerniente a la actividad diferencial de estos últimos. Tal variabilidad puede ser inter o intraespecifica e intraindividual 2, 5-8, 10, 14-16, 21, 25 .

Atento a la importancia económica de Prochilodus lineatus, el objetivo del presente trabajo fue establecer la estructura cromosómica de esta especie en ejemplares provenientes del río Paraná en la Provincia de Corrientes (Argentina), dando mayor énfasis al análisis de las regiones organizadoras de nucléolos.

\section{MATERIAL Y MÉTODOS}

Se analizaron 34 especímenes de $P$. lineatus colectados en el río Paraná, en la Provincia de Corrientes (Argentina). Antes del sacrificio se anestesió a los animales con metanosulfonato de tricaina MS-222 (Finquel $($ ). De los 34 animales estudiados, solo 7 ejemplares (3 hembras y 4 machos) arrojaron resultados satisfactorios para la coloración con nitrato de plata.

Para el estudio de los cromosomas se utilizó la porción anterior del riñón, de acuerdo con la metodología propuesta por especialistas en el tema ${ }^{22}$. Los cromosomas mitóticos se obtuvieron a través de una técnica propuesta por otros investigadores ${ }^{9}$. Las regiones organizadoras de nucleolo (NOR) se identificaron por tinción con nitrato de plata (Ag-NOR) según el método convencional ${ }^{13}$.

Las metafases fueron fotografiadas con Infinity 1 Digital Kit acoplado al microscopio óptico Olympus $B X 41$, posteriormente fueron analizadas con el programa analizador de imágenes Image ProPlus 5.1. En el cariotipo se distribuyeron los pares homólogos en orden decreciente de tamaño. Los cromosomas se clasificaron como metacéntricos (M) y submetacéntricos (SM) de acuerdo a su morfología y relación de brazos ${ }^{19}$.

\section{RESULTADOS Y DISCUSIÓN}

El análisis citogenético de la especie reveló la presencia de 54 cromosomas (meta/submetacéntricos), número fundamental igual a 108 en ambos sexos. No se detectó heteromorfismo cromosómico sexual (Figura 1A). La familia Prochilodontidae es considerada como conservativa desde el punto de vista de la macroestructura cariotípica, especialmente las especies del género Prochilodus (P. vimboides, $P$. lineatus, $P$. affinis, $P$. marggravii, $P$. cearensis, $P$. argenteus y $P$. nigricans) que poseen un cariotipo constituido por 54 cromosomas bibraquiales ${ }^{23,24}$.

También se observó la presencia de 0-2 microcromosomas $\mathrm{B}$, los cuales variaron en número inter $\mathrm{e}$ intra-individualmente en el complemento cariotípico (Figura 1A). La ocurrencia de cromosomas supernumerarios o B en $P$. lineatus provenientes de diferentes puntos de muestreo del río Paraná, estudiados en Brasil por varios autores, alcanzaron en algunas poblaciones un número que varió de 0-7 1,3,4, 20,23,24.
A través de la coloración con nitrato de plata se evidenció la NOR en posición intersticial en el brazo largo de un par de cromosomas metacéntricos, identificado como el segundo del complemento, coincidiendo con los resultados de otros autores ${ }^{24}$. En esta población los especímenes presentaron variaciones inter e intra-individual en relación al posición y tamaño de las NORs: a) la NOR se presenta única y de igual tamaño en los dos homólogos, b) NOR grande ocupando la mitad del brazo largo y única en uno de los cromosomas; pequeña y única en el otro, c) doble región NOR en uno de los cromosomas y NOR única y pequeña en su homólogo (Figura 1B a-b-c).

Las regiones organizadoras de nucléolos presentan una naturaleza polimórfica bastante evidente en peces ${ }^{8}$, tal afirmación ha sido confirmado por estu-

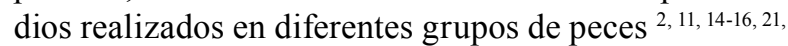
${ }^{25}$. Se ha propuesto que la diferencia de tamaño de las NORs es el resultado de alteraciones cromosómicas estructurales como duplicación, deleción y crossing-over desigual ${ }^{12}$. Estudios previos indican que probablemente la doble NOR es la consecuencia de una inversión paracéntrica ${ }^{21}$.

Los datos obtenidos en el presente trabajo y las descripciones realizadas por otros autores revelan que Prochilodus lineatus presenta un polimorfismo estructural intra e inter-individual relacionado con el tamaño, posición y números de las regiones organizadoras de nucléolos. Estos resultados contribuyen a una mejor comprensión de la organización genómica de Prochilodus lineatus y pueden ser utilizados en futuros estudios citotaxonómicos dentro de la familia Prochilodontidae.

\section{REFERENCIAS}

1. Artoni RF, Vicari MR, Endler AL, Cavallaro ZI, Jesus CM, Almeida MC, Moreira Filho O, Bertollo LA. 2006. Banding pattern of A and B chromosomes of Prochilodus lineatus (Characiformes, Prochilodontidae), with comments on B chromosomes evolution. Genetica 127: 277284.

2. Bertollo LA, Cavallaro ZI. 1992. A highly differentiated ZZ/ZW sex chromosome system in a Characidae fish, Triportheus guentheri. Cytogen Cell Genet 60: 60-63.

3. Carvalho RA, Martins-Santos IC, Dias AL. 2008. B chromosomes: an update about their occurrence in freshwater neotropical fishes (Teleostei). J Fish Biol 72: 19071932.

4. Cavallaro ZI. 1992. Estudos comparativos sobre os cromossomos B de Prochilodus scrofa Steindachner, 1881 (Pisces, Prochilodontidae) de diferentes localidades. Master Dissertation, Anais Universidade Federal de São Carlos, São Paulo, p. 32.

5. Cestari MM, Galetti PM. 1992. Chromosome studies of the Serrasalmus spilopleura (Characidae, Serrasalminae) from the Paraná-Paraguay rivers: evolutionary and cytotaxonomic considerations. Copeia 1: 108-112.

6. Cestari MM, Galetti M. 1992. Chromosome evolution in the genus Serrasalmus and cytotaxonomic considerations 
about Serrasalminae (Characidae, Pisces). Braz J Genet 15: 555-567.

7. Feldberg E, Porto JI, Bertollo LA. 1992. Karyotype evolution in Curimatidae (Teleostei, Characiformes) of the Amazon region. I. Studies on the genera Curimata, Psectrogaster, Steindachnerina and Curimatella. Braz J Genet 15: 369-383.

8. Foresti F, Almeida LF, Toledo AS. 1981. Polymorphic nature of nucleolus organizer regions in fishes. Cytogenet Cell Genet 31: 137-144.

9. Foresti F, Oliveira C, Almeida LF. 1993. A method for chromosome preparations from large fish specimens using in vitro short-term treatment with colchicine. Experientia 49: 810-813.

10. Galetti PM. 1998. Chromosome diversity in neotropical fishes: NOR studies. Italian J Zool 65: 53-56.

11. Galetti PM, Mestriner CA, Monaco PJ, Rasch EM. 1995. Post-zygotic modifications and intra- and interindividual nucleolar organizing region variations in fish: report of a case involving Leporinus friderici. Chrom Res 3: 285-290.

12. Gold JR, Li C, Shipley NS, Powers PK. 1990. Improved methods for working with chromosomes with a review of metaphase chromosome banding. J Fish Biol 37: 563-575.

13. Howell WM, Black DA. 1980. Controlled silver-staining of nucleolus organizer regions with a protective colloidal developer: a 1-step method. Experientia 36: 1014-1015.

14. Jesus CM, Moreira-Filho O. 2000. Cytogenetics studies in some Apareiodon species (Pisces, Parodontidae). Cytologia 65: 397-402.

15. Jesus CM, Moreira-Filho O. 2003. Chromosomal location of 5S and 18S rRNA genes in Prochilodus lineatus (Characiformes, Prochilodontidae). Caryologia 56: 281287.

16. Jorge LC, Moreira Filho O. 2004. Nucleolar organizer regions as markers of chromosomal polymorphism in Apareiodon affinis (Pisces, Parodontidae). Caryologia 57: 195-199.
17. Leccia MF. 1972. Consideraciones sobre la sistemática de la familia Prochilodontidae (Osteichthyes, Cypriniformes), con una sinopsis de las especies de Venezuela. Acta Biol Venez 8: 35-96.

18. Lessi E. 1968. Aspectos químicos bromatológicos de Curimbatá Prochilodus scrofa: estudo da fração protéica. Rev Faculd Odontol Araraquara 2: 121-132.

19. Levan A, Fredga K, Sandberg AA. 1964. Nomenclature for centromeric position on chromosomes. Hereditas 52 : 201-220.

20. Maistro EL, Oliveira C, Foresti F. 2000. Cytogenetic analysis of A- and B- chromosomes of Prochilodus lineatus (Teleostei, Prochilodontidae) using different restriction enzyme banding and staining methods. Genetica 108 : 119-125.

21. Moreira-Filho O, Bertollo LA, Galetti PM. 1984. Structure and variability of nucleolar organizer regions in Parodontidae fish. Can J Genet Cytol 26: 564-568.

22. Moreira-Filho O, Bertollo LA. 1991. Extration and use of the cephalic kidney for chromosome studies in small fish. Braz J Genet 14: 1085-1090.

23. Pauls E, Bertollo LA. 1983. Evidence for a system of supernumerary chromosomes in Prochilodus scrofa Steindachner, 1881 (Pisces, Prochilodontidae). Caryologia 36: 307-314.

24. Pauls E, Bertollo LA. 1990. Distribution of a supernumerary chromosomes system and aspects of karyotypic evolution in the genus Prochilodus (Pisces, Prochilodontidae). Genetica 81: 117-123.

25. Vicari MR, Almeida MC, Bertollo LA, Moreira Filho O, Artoni RF. 2006. Cytogenetic analysis and chromosomal characteristics of the polymorphic 18S rDNA in the fish Prochilodus lineatus (Characiformes, Prochilodontidae). Genet Molec Biol 29: 621-625. 